\title{
Avaliação do aleitamento materno em crianças até dois anos assistidas na atenção básica do Recife, Pernambuco, Brasil
}

\author{
Breastfeeding assessment in children up to 2 years of age assisted \\ in primary health care of Recife in the state of Pernambuco, Brazil
}

Eryka Maria dos Santos ${ }^{1}$

Luana Sales da Silva ${ }^{1}$

Bianca Fabrízzia de Sá Rodrigues ${ }^{1}$

Thalita Milena Araújo Xavier de Amorim ${ }^{1}$

Catarine Santos da Silva ${ }^{1}$

Juliana Maria Carrazzone Borba ${ }^{1}$

Fernanda Cristina de Lima Pinto Tavares ${ }^{1}$

\footnotetext{
${ }^{1}$ Centro de Ciências da Saúde, Universidade Federal de Pernambuco. Av. da Engenharia, Cidade Universitária. 50670420 Recife PE Brasil. erykasantos.nutri@

gmail.com
}

\begin{abstract}
The scope of this study was to evaluate exclusive and total breastfeeding in children up to 2 years of age treated in Basic Health Units (BHU) of Recife in the state of Pernambuco. A cross-sectional descriptive study was conducted in the Basic Health Units of Mustardinha, Jardim Uchôa, Fernandes Figueira and Upinha Novo Prado. The study population consisted of children of 0 to 24 months of age. One hundred and forty-one children from 0 to 24 months of age were evaluated, of which $56.4 \%$ were female, $22 \%$ were $\leq 6$ months old, 21.3\% were between 7 and 12 months of age and $56.7 \%$ were between 13 and 24 months of age. Analysis of the prevalence of exclusive and total breastfeeding revealed an average of 60.84 and 182.52 days, respectively. Male children, the use of pacifiers and bottle-feeding was associated with shorter exclusive breastfeeding duration.
\end{abstract}

Key words Child, Mother, Breastfeeding, Primary Health Care
Resumo O presente estudo teve por objetivo avaliar o aleitamento materno exclusivo (AME) e total em crianças até 2 anos de idade atendidas em Unidades Básicas de Saúde (UBS) do Recife-PE. Estudo descritivo transversal, realizado nas Unidades Básicas de Saúde da Mustardinha, Jardim Uchôa, Fernandes Figueira e Upinha Novo Prado. A população foi composta por crianças de 0 a 24 meses de idade. Foram avaliadas 141 crianças, das quais $54,6 \%$ eram do sexo feminino, $22 \%$ tinham idade $\leq 6$ meses, $21,3 \%$ entre 7 a 12 meses e $56,7 \%$ entre 13 e 24 meses. Ao analisar a prevalência de aleitamento materno exclusivo e total, verificou-se a mediana de 60,84 e 182,52 dias, respectivamente. Crianças do sexo masculino, uso de chupeta e mamadeira foram associados ao menor tempo de aleitamento materno exclusivo.

Palavras-chave Criança, Mãe, Aleitamento materno, Atenção primária à saúde 


\section{Introdução}

A recomendação da Organização Mundial da Saúde (OMS) é que o aleitamento materno (AM) seja exclusivo até os 6 meses de idade e complementado até 24 meses ou mais. Essa recomendação tem sido pautada em vários trabalhos que mostram benefícios dessa prática para a saúde da mulher e da criança $a^{1-3}$.

$\mathrm{O}$ fato das crianças nascerem com o sistema imunológico e gastrointestinal imaturos faz com que a introdução precoce de outros alimentos antes dos 6 meses de idade aumente os riscos de problemas digestivos, respiratórios e renais, além de interferir de forma negativa na formação dos hábitos alimentares.

Apesar de serem largamente conhecidos os benefícios do AM para a criança e para a mãe, e da existência de leis e programas de promoção, proteção e apoio a essa prática, os índices mundiais de AM ainda se encontram abaixo do recomendado ${ }^{5}$. Estudos mostram a baixa prevalência de AM a nível regional, nacional e mundial e destacam inúmeros fatores que podem influenciar esta prática ${ }^{2,6,7}$. Assim, o acompanhamento permanente e a vigilância continuada tornam-se imprescindíveis para a consolidação da promoção, da proteção e do apoio ao AM, a fim de que as taxas de morbimortalidade infantil possam continuar sendo reduzidas.

No final da década de 2000, as ações de promoção, proteção e apoio ao AM passaram a ser desenvolvidas a partir de um ou mais dos eixos prioritários, que incluem: o eixo da atenção básica, da atenção hospitalar, da rede brasileira de bancos de leite humano, da proteção legal ao AM, da mobilização social, do monitoramento dos indicadores de $\mathrm{AM}^{8}$.

Neste contexto, os estudos que acompanham a prática do AM e avaliam os fatores que a influenciam são importantes para subsidiar iniciativas pontuais para o fortalecimento de ações que contribuem para melhorar o quadro do AM no Brasil. Apesar das inúmeras pesquisas realizadas nessa temática, destaca-se a necessidade de se avaliar essa prevalência e seus fatores de influência na população atendida pelo Sistema Único de Saúde (SUS), em especial na atenção primária, uma vez que geralmente se trata de uma população com piores condições socioeconômicas e está formalmente assistida pela saúde pública, fatores a serem considerados na articulação de ações que visem proteger e promover o AM.

A Estratégia Saúde da Família (ESF) corresponde a um espaço amplo para a promoção, proteção e apoio ao AM na atenção básica. A operacionalização de ações nas Unidades Básicas de Saúde deve envolver toda a equipe de saúde, além de poder contar, eventualmente, com parcerias estabelecidas com Instituições de Ensino e Pesquisa ${ }^{8}$.

O presente estudo teve por objetivo avaliar o AM das crianças de até dois anos de idade atendidas em Unidades Básicas de Saúde do Distrito V, Recife - PE.

\section{Método}

A população em estudo foi composta por crianças de até 2 anos de idade acompanhadas em 4 Unidades Básica de Saúde (UBS) do Distrito Sanitário V da Cidade do Recife - PE, vinculadas ao Programa de Educação para o Trabalho em Saúde (PET-SAÚDE), sendo elas: UBS Mustardinha, UBS Fernandes Figueira, UBS Jardim Uchôa e Upinha Novo Prado. Foi realizado um levantamento prévio e identificou-se que cada UBS acompanhava cerca de 100 crianças de até dois anos (universo de 400 crianças). Para o cálculo da amostra foi considerado uma estimativa de $38 \%$ de aleitamento materno exclusivo (AME) até o $6^{\circ}$ mês de vida, baseada na prevalência de AME encontrada na cidade do Recife na II Pesquisa de Prevalência de Aleitamento Materno nas Capitais Brasileiras e Distrito Federal ${ }^{2}$, um erro de $7 \%$, perdas de $10 \%$ e nível de confiança de $95 \%$, o que resultou em uma amostra de 135 indivíduos, os quais se procurou distribuir igualmente nas quatro UBS. O critério de inclusão foi o cadastramento da criança na UBS e a seleção foi feita por meio de sorteio e pulo amostral a partir da lista dos Agentes Comunitários de Saúde (ACS). Inicialmente foi sorteado o número 5 da lista, considerando um pulo amostral de 3 .

Os dados foram coletados a partir da aplicação de questionários realizados com as mães ou cuidadores das crianças no período de novembro/2014 a fevereiro/2015. Estas eram visitadas em domicílio junto com o ACS responsável pela área ou contactadas durante a consulta da puericultura. Aquelas em que não se obtinha êxito no contato eram substituídas pela próxima criança na lista de cadastro da UBS. O questionário aplicado foi baseado no módulo de questões materno-infantil utilizado na II e na III Pesquisa Estadual de Saúde e Nutrição do Estado de Pernambuco (1997 e 2006) $)^{9}$, contemplando as seguintes variáveis:

- Mãe: idade; escolaridade; número de consultas pré-natal; que profissionais a acompanhou; 
recebeu orientação sobre aleitamento materno (individual ou em grupo); qual profissional fez a orientação; tipo do parto; tempo de licença maternidade; ocupação materna; renda da família; número de moradores.

- Criança: sexo; idade; tempo de aleitamento materno exclusivo e total; uso de chupeta ou mamadeira.

Os questionários foram aplicados por duas discentes do curso de graduação em nutrição da UFPE, as quais passaram por um treinamento prévio de 20 horas, seguido por testagem do instrumento e logística de campo com 10 mães/ cuidadoras de crianças não sorteadas da UBS Mustardinha.

Os dados foram digitados em dupla entrada no software Epi Info versão 3.5.1 para Windows e analisados no software SPSS versão 12. Foram realizadas estatísticas descritivas e o teste do quiquadrado de Pearson, considerando-se nível de significância quando $\mathrm{p}<0,05$.

O projeto foi aprovado pelo Comitê de Ética em Pesquisa com Seres Humanos (CEP) do Centro de Ciências da Saúde da Universidade Federal de Pernambuco, seguindo as orientações da Resolução no 466/12 do Conselho Nacional de Saúde. Participaram do estudo as mães que concordaram e assinaram o Termo de Consentimento Livre e Esclarecido (TCLE).

\section{Resultados}

Foram realizadas 141 entrevistas, sendo 36 na UBS Mustardinha, 35 na UBS Fernandes Figueira, 35 na UBS Jardim Uchôa e 35 na Upinha Novo Prado. Os dados coletados referem-se às condições sócio-demográficas da família, dados da criança, dados obstétricos, relacionados ao pré-natal e AM.

$\mathrm{Na}$ análise dos resultados, é possível observar que a maioria das mães encontrava-se na faixa etária de 20-35 anos (71,6\%), sendo predominante a quantidade de mães que não trabalham fora de casa (72,3\%). Quanto ao tempo de licença maternidade, $80,9 \%$ não trabalhavam fora na época da gestação ou tinham emprego informal. Em relação ao estado civil, o mais citado foi a união estável $(48,9 \%)$ e quanto a escolaridade materna, $62,1 \%$ relataram ter cursado o ensino médio ou mais. A maior parte $(73,6 \%)$ tinha renda per capita $<0,5$ salário mínimo (Tabela 1).

Com relação ao número de filhos e tipo de parto, 51,1\% afirmaram que a criança em estudo era o primeiro filho e $53,9 \%$ destes nasceram de
Tabela 1. Variáveis relacionadas às condições socioeconômicas e obstétricas das mães e das crianças atendidas em Unidades Básicas de Saúde. Recife-PE, 20142015.

\begin{tabular}{|c|c|c|}
\hline Variáveis & $\begin{array}{c}\text { Número } \\
(\mathrm{N})\end{array}$ & $\begin{array}{c}\text { Freqüência } \\
(\%)\end{array}$ \\
\hline \multicolumn{3}{|l|}{ Idade Materna * } \\
\hline$\leq 19$ anos & 26 & 20,5 \\
\hline $20-35$ anos & 91 & 71,6 \\
\hline$\geq 36$ anos & 10 & 7,9 \\
\hline \multicolumn{3}{|l|}{ Profissão Atual } \\
\hline Do lar & 102 & 72,3 \\
\hline Emprego formal & 28 & 19,9 \\
\hline Emprego Informal/Autônomo & 11 & 7,8 \\
\hline \multicolumn{3}{|l|}{ Tempo de Licença Maternidade } \\
\hline $\begin{array}{l}\text { Não trabalhava fora/ emprego } \\
\text { informal }\end{array}$ & 114 & 80,9 \\
\hline 3 meses & 01 & 0,7 \\
\hline 6 meses & 02 & 1,4 \\
\hline \multicolumn{3}{|l|}{ Estado Civil } \\
\hline Solteira & 36 & 25,5 \\
\hline Casada & 33 & 23,4 \\
\hline União Estável & 69 & 48,9 \\
\hline Divorciada & 03 & 2,1 \\
\hline \multicolumn{3}{|l|}{ Nível de Escolaridade ${ }^{* *}$} \\
\hline Nenhum até $4^{\text {a }}$ série & 11 & 7,9 \\
\hline $5^{a}$ a $8^{a}$ série & 42 & 30,0 \\
\hline Ensino médio ou mais & 87 & 62,1 \\
\hline \multicolumn{3}{|l|}{ Renda per capita ${ }^{\star * \star}$} \\
\hline$<0,5$ salário mínimo & 95 & 73,6 \\
\hline 0,5 - 0,99 salário mínimo & 28 & 21,7 \\
\hline \multicolumn{3}{|l|}{ Número de Filhos } \\
\hline 1 & 72 & 51,1 \\
\hline 2 & 40 & 28,4 \\
\hline 3 & 15 & 10,6 \\
\hline 4 ou mais & 14 & 9,9 \\
\hline \multicolumn{3}{|l|}{ Tipo de Parto } \\
\hline Vaginal & 76 & 53,9 \\
\hline Cesáreo & 65 & 46,1 \\
\hline \multicolumn{3}{|l|}{ Idade Gestacional $* * * *$} \\
\hline Pré-termo & 11 & 8,0 \\
\hline A termo & 127 & 92,0 \\
\hline
\end{tabular}

parto vaginal. Em se tratando da idade gestacional, 92\% das crianças nasceram a termo, sendo a mediana de 40 semanas. Quanto ao pré-natal, $74,3 \%$ iniciaram ainda no $1^{\circ}$ trimestre e $63,1 \%$ realizaram 6 consultas ou mais. No que diz respeito à idade e sexo da criança, 56,7\% tinham entre 13 e 24 meses e 54,6\% eram do sexo feminino. Das 141 crianças participantes do estudo, 52,5\% 
Tabela 1. Variáveis relacionadas às condições socioeconômicas e obstétricas das mães e das crianças atendidas em Unidades Básicas de Saúde. Recife-PE, 20142015.

\begin{tabular}{|c|c|c|}
\hline Variáveis & $\begin{array}{l}\text { Número } \\
(\mathrm{N})\end{array}$ & $\begin{array}{l}\text { Freqüência } \\
(\%)\end{array}$ \\
\hline \multicolumn{3}{|c|}{ Mês de início do pré-natal $* * * \star$} \\
\hline Não fez & 01 & 0,7 \\
\hline $1-3$ meses & 104 & 74,3 \\
\hline $4-6$ meses & 30 & 21,4 \\
\hline 7 meses ou mais & 05 & 3,6 \\
\hline \multicolumn{3}{|l|}{ No de consultas realizadas } \\
\hline 0 & 01 & 0,7 \\
\hline $1-5$ & 20 & 14,1 \\
\hline 6 ou mais & 89 & 63,1 \\
\hline Não sabe/Não lembra & 31 & 22,0 \\
\hline \multicolumn{3}{|l|}{ Idade da criança } \\
\hline$\leq 6$ meses & 31 & 22,0 \\
\hline 7 - 12 meses & 30 & 21,3 \\
\hline $13-24$ meses & 80 & 56,7 \\
\hline \multicolumn{3}{|l|}{ Sexo da criança } \\
\hline Masculino & 64 & 45,4 \\
\hline Feminino & 77 & 54,6 \\
\hline \multicolumn{3}{|l|}{ Uso de chupeta } \\
\hline Sim & 74 & 52,5 \\
\hline Não & 67 & 47,5 \\
\hline \multicolumn{3}{|l|}{ Uso de mamadeirat**** } \\
\hline Não usa & 24 & 17,1 \\
\hline Crianças $\leq 6$ meses & 28 & 20,0 \\
\hline Crianças $7-12$ meses & 24 & 17,1 \\
\hline Crianças $13-24$ meses & 64 & 45,7 \\
\hline $\begin{array}{l}{ }_{* * *} 14 \text { casos sem informação } \\
* * * * * \\
1 \text { casos sem informação }\end{array}$ & $\begin{array}{r}* * 1 \text { caso se } \\
* * * * 3 \text { casos } s\end{array}$ & $\begin{array}{l}\mathrm{m} \text { informação } \\
\mathrm{m} \text { informação }\end{array}$ \\
\hline
\end{tabular}

usavam chupeta, com mediana do tempo de chupeta de 15 dias (IQ = 3-60). Em relação ao uso de mamadeira, verificou-se que a maior parte das crianças utilizava $(82,8 \%)$ (Tabela 1$)$.

Dados sobre o pré-natal e consultas após o nascimento mostram que as mães, em muitos casos, eram acompanhadas por mais de um profissional de saúde, sendo o profissional de enfermagem o mais citado, no pré-natal (73\%) e após o nascimento $(69,5 \%)$. Dessas mães, 95\% afirmaram ter recebido algum tipo de orientação sobre o AM, seja de forma individual, em grupo ou ambos, e $48,1 \%$ disseram ter sido no período prénatal, enquanto que $39,1 \%$ receberam tanto no pré-natal quanto após o nascimento da criança. Quanto ao local onde receberam orientações sobre AM, os mais citados foram USF e/ou mater- nidade. Em relação ao profissional que orientou, o enfermeiro (a) foi o profissional mais atuante. Quando questionadas, a maioria afirmou ter recebido informações-chave sobre a amamentação (Tabela 2).

Os questionamentos às mães quanto aos benefícios que elas acreditavam que o leite materno poderia trazer tanto para ela como para a saúde da criança mostraram que em todas as opções a maior parte das mães respondeu que sabiam os benefícios do AM. Em relação ao tempo em que o leite materno deve ser dado pela primeira vez, $85,1 \%$ responderam logo após o parto, em até 30 minutos e $67,4 \%$ responderam que o leite materno deve ser oferecido sempre que o bebê solicitar (Tabela 3).

Quanto ao fato da alimentação interferir ou não na qualidade do leite, $89,4 \%$ responderam que sim. Quando questionadas quanto à importância da alimentação de forma geral, grande parte relatou achar importante o consumo de legumes/verduras, frutas, carnes, leite e massas. Quantidade considerável de mães (58,2\%) respondeu que o doce também era importante. A pergunta seguinte foi se existia algo que poderia influenciar a produção do leite e 96,5\% responderam que sim. A alimentação, aumento do consumo de líquidos, o estímulo da sucção, massagem das mamas, tranqüilidade da mãe, assim como doce foram fatores relacionados a esse aumento. Em se tratando do conhecimento das mães sobre o fato de existirem ou não situações que podem impedir ou dificultar o aleitamento materno, 83,7\% disseram que sim. Os fatores mais relacionados a essas dificuldades foram: problema nas mamas, mastite, doença e uso de medicamentos pela mãe. Dentre as que citaram outros, muitas relataram o estresse como fator de dificuldade (Tabela 3).

Os dados relacionados ao tempo de aleitamento materno total (AMT) e exclusivo (AME) mostraram que a mediana do AMT foi de 182,52 dias e AME de 60,84 dias (Gráfico 1). Importante ressaltar que dentre as 141 crianças estudadas, 7 nunca foram amamentadas, 24 nunca mamaram exclusivamente, 59 crianças ainda mamam e apenas 2 ainda estavam em aleitamento materno exclusivo. Não houve diferença estatisticamente significante do tempo de AME com relação à idade materna, escolaridade, renda, trabalho da mãe, estado civil e tipo de parto. Quanto a UBS, também não foi vista diferença estatisticamente significante, entretanto observa-se maior prevalência de AME na UBS Jardim Uchôa em relação às demais. O sexo masculino, o uso de chupeta e 
Tabela 2. Dados sobre pré-natal e consultas após o nascimento de crianças até 2 anos atendidas em Unidades Básica de Saúde. Recife-PE, 2014-2015.

\begin{tabular}{|c|c|c|}
\hline & $\begin{array}{l}\text { Número } \\
(\mathrm{N})\end{array}$ & $\begin{array}{c}\text { Freqüência } \\
(\%)\end{array}$ \\
\hline \multicolumn{3}{|c|}{ Quem acompanhou o Pré-natal? } \\
\hline Enfermeiro & 103 & 73,0 \\
\hline Médico & 61 & 43,3 \\
\hline ACS & 78 & 55,3 \\
\hline
\end{tabular}

Quem acompanha nas consultas após o nascimento?

$\begin{array}{lll}\text { Enfermeiro } & 98 & 69,5 \\ \text { Médico } & 57 & 40,4 \\ \text { ACS } & 92 & 65,2\end{array}$

Recebeu orientação sobre AM?

\begin{tabular}{|c|c|c|}
\hline Sim, individual & 78 & 55,3 \\
\hline Sim, em grupo & 33 & 23,4 \\
\hline $\begin{array}{l}\text { Sim, individual e em } \\
\text { grupo }\end{array}$ & 23 & 16,3 \\
\hline Não & 07 & 5,0 \\
\hline \multicolumn{3}{|l|}{ Se sim, quando?* } \\
\hline No pré-natal & 64 & 48,1 \\
\hline $\begin{array}{l}\text { Consultas após o } \\
\text { nascimento }\end{array}$ & 02 & 1,5 \\
\hline Maternidade & 15 & 11,3 \\
\hline $\begin{array}{l}\text { No pré-natal e após o } \\
\text { nascimento }\end{array}$ & 52 & 39,1 \\
\hline \multicolumn{3}{|l|}{ Onde ${ }^{*}$} \\
\hline Unidade Saúde da Família & 38 & 28,6 \\
\hline Maternidade & 41 & 30,8 \\
\hline USF + Maternidade & 44 & 33,1 \\
\hline Outros $^{\mathrm{a}}$ & 10 & 7,5 \\
\hline
\end{tabular}

Quem orientou?**

$\begin{array}{llr}\text { Nutricionista } & 12 & 9,1 \\ \text { Enfermeiro } & 57 & 43,2 \\ \text { Médico } & 22 & 16,6 \\ \text { ACS + outro(s) } & 41 & 31,1\end{array}$

profissional(is)

Que informações recebeu?

Amamentar de forma

exclusiva até os 6 meses

$127 \quad 94,7$

O leite materno protege

contra doenças

Como cuidar das mamas $\quad 118 \quad 88,1$

Posição para amamentar $\quad 118 \quad 88,1$

Pega correta

122

91,0

${ }^{*} 1$ caso sem informação ${ }^{*} 2$ casos sem informação

${ }^{\mathrm{a}}$ Outros $=2$ ou mais opções entre Maternidade, Banco de Leite, USF, Hospital Privado e Consultório Particular.
Tabela 3. Concepção de mães de crianças até 2 anos atendidas em Unidades Básica de Saúde quanto aos benefícios, momento, intervalo de tempo ideal para o aleitamento materno, a importância da alimentação e fatores que auxiliam ou prejudicam esta prática. Recife-PE, 2014-2015.

\begin{tabular}{|c|c|c|}
\hline & Número $(\mathrm{N})$ & Freqüência (\%) \\
\hline \multicolumn{3}{|c|}{ Benefícios que o aleitamento materno traz: ${ }^{*}$} \\
\hline Protege contra doenças & 132 & 93,6 \\
\hline Fortalece o vínculo & 134 & 95,0 \\
\hline
\end{tabular}

afetivo

$\begin{array}{lll}\text { É um alimento completo } & 119 & 84,4 \\ \text { É de fácil digestão } & 127 & 90,1 \\ \text { O bebê cresce saudável } & 134 & 95,0\end{array}$

Momento o leite materno deve ser oferecido ao bebê pela primeira vez:

$\begin{array}{lcc}\begin{array}{l}\text { Logo após o parto (até } \\ \text { 30min) }\end{array} & 120 & 85,1 \\ \begin{array}{l}\text { De } 30 \mathrm{~min} \text { até } 1 \mathrm{~h} \text { após o } \\ \text { parto }\end{array} & 13 & 9,2 \\ \begin{array}{l}\text { De } 1 \mathrm{~h} \text { até } 2 \mathrm{~h} \text { após o } \\ \text { parto }\end{array} & 08 & 5,7\end{array}$

Intervalo de tempo que o leite materno deve ser oferecido para o Recém-Nascido (RN):

$\begin{array}{llr}\begin{array}{l}\text { Sempre que o RN } \\ \text { solicitar }\end{array} & 95 & 67,4 \\ \text { A cada 3h } & 30 & 21,3 \\ \text { A cada 2h } & 08 & 5,7 \\ \text { A cada hora } & 06 & 4,3 \\ \text { Não sabe/Outro } & 02 & 1,4\end{array}$

A alimentação da mãe interfere na qualidade do leite?

$\begin{array}{lrr}\text { Sim } & 126 & 89,4 \\ \text { Não } & 14 & 9,9 \\ \text { Não sabe } & 01 & 0,7\end{array}$

Tipos de alimentos importantes consumir: *

$\begin{array}{lrl}\text { Legumes/verduras } & 135 & 95,7 \\ \text { Frutas } & 139 & 98,6 \\ \text { Carnes/aves/peixes } & 137 & 97,2 \\ \text { Leite e derivados } & 133 & 94,3 \\ \text { Massas } & 91 & 64,5 \\ \text { Doces } & 82 & 58,2 \\ \text { Frituras } & 17 & 12,1\end{array}$

Existe alguma forma de estimular a produção de leite?

$\begin{array}{lrr}\text { Sim } & 136 & 96,5 \\ \text { Não } & 05 & 3,5\end{array}$

O que pode estimular a produção do leite? *

\begin{tabular}{lrr} 
Alimentação & 119 & 84,4 \\
Aumentar consumo de & 126 & 89,4 \\
líquidos & & \\
Massagem nas mamas & 111 & 78,7 \\
Tranqüilidade da mãe & 119 & 84,4 \\
Outros & 6 & 4,3 \\
\hline
\end{tabular}

mamadeira mostrou-se associado à menor prevalência de AME (Tabela 4). 
Tabela 3. Concepção de mães de crianças até 2 anos atendidas em Unidades Básica de Saúde quanto aos benefícios, momento, intervalo de tempo ideal para o aleitamento materno, a importância da alimentação e fatores que auxiliam ou prejudicam esta prática. Recife-PE, 2014-2015.

\begin{tabular}{lcc} 
& Número (N) & Freqüência (\%) \\
\hline $\begin{array}{l}\text { Existe algo que pode } \\
\text { impedir o aleitamento? }\end{array}$ & & \\
$\quad$ Sim & 118 & 83,7 \\
$\quad$ Não & 23 & 16,3 \\
Que situação? & & \\
$\quad$ Problema nas mamas & 108 & 76,6 \\
$\quad$ Mastite & 103 & 73,0 \\
Doença da mãe & 108 & 76,6 \\
$\quad$ Uso de medicamentos & 107 & 75,9 \\
pela mãe & & \\
Outros & 31 & 22,0 \\
\hline
\end{tabular}

* Questões que as mulheres poderiam escolher mais de uma opção como resposta. ridade psicológica ${ }^{11}$. No presente estudo, não foi encontrada diferença estatística relacionada à idade materna e tempo de AME, corroborando com Guimarães et al. ${ }^{12}$. No entanto, observa-se uma tendência às mães mais novas amamentarem por um período de tempo menor.

As modificações socioculturais ocorridas nos últimos anos fazem com que a mulher torne-se cada vez mais inserida no mercado de trabalho e participativa nas despesas familiares, o que pode ter influência na prática do $\mathrm{AM}$, determinando sua diminuição ou fim e contribuindo para aumento da morbimortalidade infantil ${ }^{13,14}$. Nesse estudo, observou-se que a maioria das mães não trabalhava fora de casa, o que poderia proporcionar mais tempo para amamentar seu filho, entretanto, nas análises estatísticas não houve diferença entre os grupos.

Damião ${ }^{15}$ observou que entre as mulheres que não trabalhavam fora de casa a prevalência de aleitamento era o dobro daquelas que trabalhavam. Isso pode ser decorrente da maior ansiedade na introdução precoce de outros alimentos a fim de adaptar a criança para o retorno ao trabalho, uma vez que há grande falta de respeito à lei que garante direito a dois intervalos de $30 \mathrm{~min}$ durante a jornada de trabalho, falta de creches nas empresas, além da falta de orientação quanto à coleta e conservação adequada do leite materno $^{16}$.

Com relação ao estado civil, 72,3\% moravam com seus companheiros, considerando-se as que informaram ser casadas ou ter união estável. Não foi encontrada diferença estatística entre estado civil e tempo de AME, no entanto, estudos mostram que o apoio do companheiro é fundamental no sucesso ou não da amamentação $0^{17}$. A relação da paternidade com a gestação e lactação pode ocasionar conflitos entre a relação marido e mulher, como afastamento do casal, sentimento de ciúme, rivalidade com o filho. A família possui papel importante na promoção do aleitamento materno, o apoio do companheiro assim como dos demais familiares auxiliam no desenvolvimento da autoconfiança e satisfação emocional da mãe ${ }^{18}$.

A maior parte das mulheres possuía um nível de escolaridade considerado alto, $62,1 \%$ com o ensino médio ou mais. O maior nível de escolaridade está relacionado à maior facilidade da mãe em assimilar as informações passadas no pré-natal, o que pode contribuir com o sucesso da amamentação. Por outro lado, maior tempo de escolaridade também contribui para inserção desta mulher no mercado de trabalho, podendo 


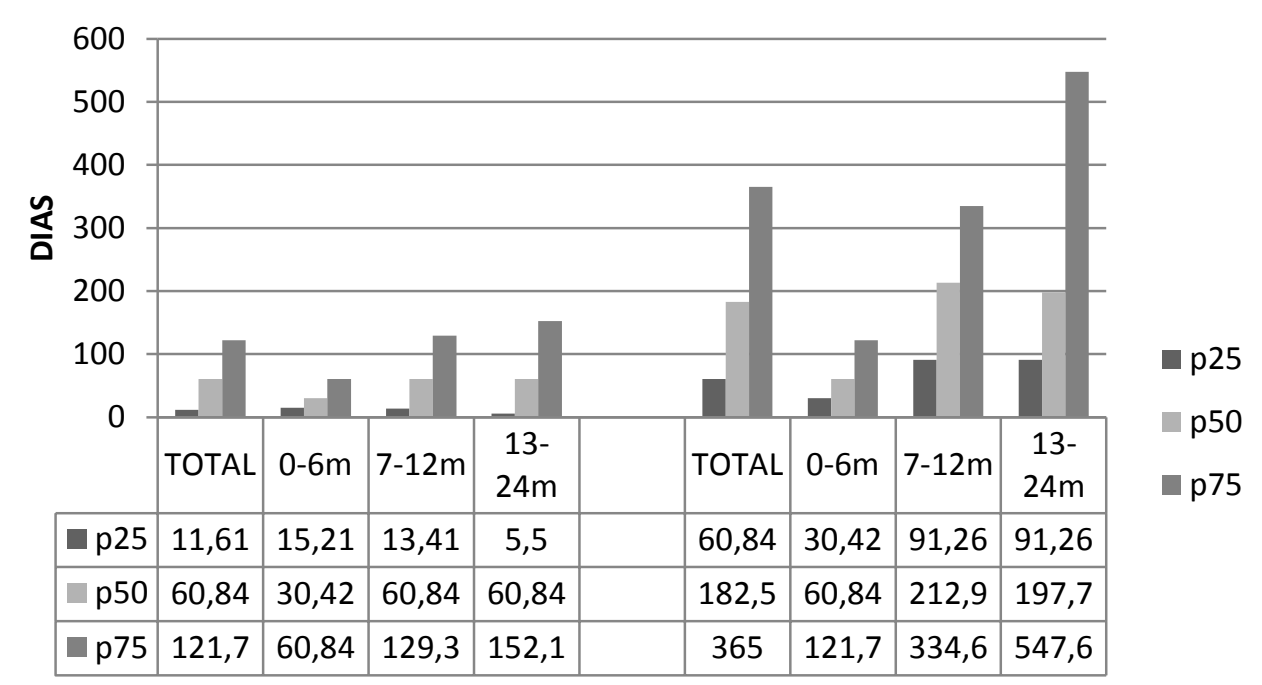

Aleitamento materno
exclusivo

Aleitamento materno total

Gráfico 1. Valores de p25, p50 e p75 em dias do tempo de aleitamento materno exclusivo (AME) e total (AMT) de crianças até 2 anos de idade atendidas em Unidades Básicas de Saúde. Recife-PE, 2014-2015.

limitar o AME. Na análise relacionada ao tempo de AME e escolaridade materna, não se observou diferença estatisticamente significante.

Segundo Machado et al. ${ }^{19}$, a prevalência de AM sofre influência direta do nível instrucional da mesma, sendo mais presente a intenção em amamentar nas mães com maior padrão de instrução. Dados contrários foram apontados por Carrascoza et al. $^{20}$, comprovando que a interrupção do AM foi predominante nas mães com maior nível.

Faleiros et al. ${ }^{21}$ realizaram uma revisão da literatura onde foi visto que nos países não industrializados as mulheres de classe baixa e média amamentavam mais do que as de classe alta. Dados semelhantes são mostrados por Wenzel e Souza ${ }^{22}$, que avaliaram prevalência de AM em diferentes regiões brasileiras e em todas elas, a maior renda foi associada a menor tempo de AM, devido provavelmente, a falta de condições das mães em adquirir outros alimentos. Os dados encontrados no presente estudo mostraram que 73,6\% tinha renda per capita $<0,5$ salário mínimo, mas não houve diferença entre os grupos de menor e maior renda e o tempo de AME. Em estudo realizado por Boff et al. $^{23}$, as mães com maior renda demonstraram maior entendimento quanto aos benefícios da amamentação.

A relação entre o nível socioeconômico e o AM é complexa e contraditória, uma vez que famílias de alto nível socioeconômico na maioria das vezes também apresentariam maior nível de instrução, o que ajudaria na compreensão da prática e benefícios do AM para mãe e bebê, mas também têm mais facilidade em adquirir substitutos do leite materno, chupeta e mamadeira. Em contrapartida, as mães de menor nível socioeconômico e provavelmente menor nível de instrução teriam maior dificuldade na assimilação das informações passadas, mas também maior dificuldade na obtenção de fórmulas infantis, chupetas e mamadeiras ${ }^{24}$.

Mediante a análise dos resultados, foi visto que em 51,1\% dos casos, a criança do estudo era o primeiro filho. A primiparidade da mãe pode estar associada ao desmame precoce. Ramos e Ramos $^{25}$ ressaltaram como fatores de influência negativa na prática do AME a falta de experiência e a questão cultural e saberes passados de geração a geração.

O tipo de parto e a idade gestacional da criança exercem influência sobre a prevalência do AM, 
Tabela 4. Relação entre tempo de aleitamento materno exclusivo e variáveis que influenciam nesta prática em crianças até 2 anos atendidas em Unidades Básica de Saúde. Recife-PE, 2014-2015.

\begin{tabular}{|c|c|c|c|c|c|}
\hline & \multicolumn{2}{|c|}{ AME $\leq 3$ MESES } & \multicolumn{2}{|c|}{ AME $\geq 4$ MESES } & \multirow[b]{2}{*}{ Valor de $\mathrm{p}^{\mathrm{a}}$} \\
\hline & $\mathbf{N}$ & $\%$ & $\mathbf{N}$ & $\%$ & \\
\hline \multicolumn{6}{|l|}{ Idade Materna* } \\
\hline$\leq 19$ anos & 20 & 87,0 & 03 & 13,0 & 0,086 \\
\hline $20-35$ anos & 51 & 64,6 & 28 & 35,4 & \\
\hline$\geq 36$ anos & 05 & 55,6 & 04 & 44,4 & \\
\hline \multicolumn{6}{|l|}{ Escolaridade Materna** } \\
\hline Nenhum até $4^{\mathrm{a}}$ série & 05 & 45,5 & 06 & 54,5 & 0,088 \\
\hline $5^{\mathrm{a}}$ a $8^{\mathrm{a}}$ série & 28 & 80,0 & 07 & 20,0 & \\
\hline Ensino médio ou mais & 55 & 70,5 & 23 & 29,5 & \\
\hline \multicolumn{6}{|l|}{ Renda per capita ${ }^{\star * \star}$} \\
\hline$<0,5$ salário mínimo & 58 & 67,4 & 28 & 32,6 & 0,378 \\
\hline$\geq 0,5$ salário mínimo & 22 & 78,6 & 06 & 21,4 & \\
\hline \multicolumn{6}{|l|}{ Profissão Materna } \\
\hline Do lar & 73 & 71,6 & 29 & 28,4 & 0,288 \\
\hline Trabalha fora & 32 & 82,1 & 07 & 17,9 & \\
\hline \multicolumn{6}{|l|}{ Estado Civil ${ }^{\star * * *}$} \\
\hline Solteira/divorciada & 20 & 64,5 & 11 & 35,5 & 0,472 \\
\hline Casada/ União estável & 69 & 73,4 & 25 & 26,6 & \\
\hline \multicolumn{6}{|l|}{ Tipo de Parto ${ }^{\star * * *}$} \\
\hline Vaginal & 47 & 71,2 & 19 & 28,8 & 1,000 \\
\hline Cesáreo & 42 & 71,2 & 17 & 28,8 & \\
\hline \multicolumn{6}{|l|}{$\mathrm{UBS}^{* * * *}$} \\
\hline Mustardinha & 22 & 68,8 & 10 & 31,2 & 0,529 \\
\hline Fernandes Figueira & 24 & 80,0 & 06 & 20,0 & \\
\hline Jardim Uchôa & 21 & 63,6 & 12 & 36,4 & \\
\hline Novo Prado & 22 & 73,3 & 08 & 26,7 & \\
\hline \multicolumn{6}{|l|}{ Sexo da criança } \\
\hline Feminino & 55 & 85,9 & 09 & 14,1 & 0,008 \\
\hline Masculino & 50 & 64,9 & 27 & 35,1 & \\
\hline \multicolumn{6}{|l|}{ Uso de Chupeta ${ }^{* * * *}$} \\
\hline Sim & 52 & 86,7 & 08 & 13,3 & 0,001 \\
\hline Não & 37 & 56,9 & 28 & 43,1 & \\
\hline \multicolumn{6}{|l|}{ Uso de mamadeira ${ }^{* * * * *}$} \\
\hline Sim & 79 & 77,5 & 23 & 22,5 & 0,002 \\
\hline Não & 09 & 40,9 & 13 & 59,1 & \\
\hline
\end{tabular}

*30 casos sem informação ${ }^{* *} 19$ casos sem informação ${ }^{* * *} 27$ casos sem informação ${ }^{* * * *} 16$ casos sem informação ${ }^{* * * *} 17$ casos sem informação ${ }^{a}$ Teste do Qui-quadrado de Pearson com correção de Yates quando necessário.

sendo o parto cesáreo e o nascimento pré-termo mais associados negativamente a esta prática. Estudo realizado em unidades de saúde de 24 países destacou o parto cesáreo como fator que influencia negativamente esta prática ${ }^{6}$. Neste estudo, observou-se que $53,9 \%$ das crianças nasceram de parto vaginal e $92 \%$ a termo. Não foi vista diferença estatística significante quanto ao AME e o tipo de parto, mas observa-se um percentual elevado de partos cesáreos realizados $(46,1 \%)$, corroborando com os achados por Souza et al. ${ }^{17}$, não condizendo com o preconizado pela OMS, que estes percentuais estejam entre $5-15 \%$ dos partos totais ${ }^{24}$. Esse fato deve alertar os profissionais de saúde quanto à correta orientação das mães sobre os riscos-benefícios dessa prática e, sempre que possível, o encorajamento ao parto vaginal, já que este é apontado como melhor promotor do AM, pois permite o contato mais precoce entre mãe-bebê, assim como a primeira mamada.

Neste estudo, $74,3 \%$ das mulheres iniciaram o pré-natal ainda no $1^{\circ}$ trimestre e $63,1 \%$ rea- 
lizaram seis ou mais consultas, demonstrando conformidade com o Programa de Humanização do Pré-Natal e Nascimento (PHPN), o qual recomenda que a gestante deva realizar no mínimo seis consultas ${ }^{26}$. Quanto mais cedo for iniciado e maior o número de consultas, maior as chances de essa mãe receber e assimilar as orientações de forma adequada e haver detecção de possíveis problemas.

Quanto à caracterização das crianças estudadas, $54,6 \%$ eram do sexo feminino. A II Pesquisa de Prevalência de Aleitamento Materno nas Capitais Brasileiras e Distrito Federal apontou o sexo do lactente como fator que influencia na prática do aleitamento, sendo o sexo feminino associado à maior prevalência ${ }^{2}$. Estudos apontam maior chance de interrupção do AM em crianças do sexo masculino ${ }^{17}$. Perez-Escamilla et al. ${ }^{27}$, num estudo realizado na América Latina sugeriu que mães de meninos imaginariam que estes precisariam de maior aporte nutricional, necessitando receber outros alimentos, além do leite materno. Corroborando com esses achados, o presente estudo mostrou que crianças do sexo feminino têm maior prevalência de tempo de AME.

Das crianças estudadas, 52,5\% faziam uso de chupeta e a mediana do tempo de introdução foi de 15 dias. Vários estudos têm mostrado associação negativa entre uso de chupeta e $\mathrm{AME}^{28}$. O uso da chupeta também pode estar relacionado às dificuldades encontradas pela mãe na amamentação, fazendo com que estas usem a chupeta como forma de acalmar a criança, refletindo insegurança da mãe diante da tarefa de cuidar do bebê. Estudos mostram que o uso da chupeta seria um marcador de desmame precoce e estaria associada à insegurança da mãe em lidar com o choro do bebêt ${ }^{29}$.

A precoce exposição a bicos artificiais, como chupeta e mamadeira, pode contribuir para o desmame precoce devido à confusão de bicos pelo bebê, dificultando a correta pega e sucção para o sucesso do AM, além de serem prejudiciais para desenvolvimento da criança ${ }^{30}$. Neu et al. ${ }^{31} \mathrm{em}$ seu estudo aponta que as crianças que receberam mamadeira por período superior a dois anos, a maioria não recebeu aleitamento materno ou o fez por um curto período. Pellegrinelli et al. ${ }^{32}$ observaram que a prevalência do AME foi menor entre as crianças que usavam chupeta $(38,4$ versus $43,2 \%$ que não faziam uso; $p<0,001)$ e mamadeira ( 13,5 versus $46,6 \%$; $<<0,001)$. Nesse caso, $52,5 \%$ das crianças usavam chupeta e $82,8 \%$ usavam mamadeira e este uso teve relação negativa com a prática do AME.
Dados sobre o acompanhamento pré-natal e nas consultas após o nascimento mostram que nos dois momentos o profissional de saúde mais citado foi o enfermeiro. Neste cenário, destacase a importância do profissional de enfermagem na promoção da prática do $\mathrm{AM}$, que neste estudo correspondeu a 73 e $69,5 \%$ no pré-natal e nas consultas pós-nascimento, respectivamente. $\mathrm{O}$ profissional de enfermagem é quem muitas vezes realiza todo pré-natal de baixo risco e tem assim, a oportunidade de desenvolver trabalho educacional no que diz respeito ao AM. Apesar dessa importante contribuição, deve-se ressaltar a necessidade de um trabalho multidisciplinar, no qual cada profissional de saúde deveria abordar os aspectos do AM de acordo com sua área, beneficiando mãe e bebê numa assistência integral ${ }^{33}$.

$O$ processo de AM deve ser considerado num contexto sociocultural e familiar, e a partir daí promover o cuidado não só à mãe e ao bebê, mas também a toda família ${ }^{34}$. Uma atenção de qualidade e de forma humanizada tanto no período pré-natal como nas fases de parto e pós-parto são fundamentais para o sucesso da amamentação, onde são identificadas na prática as maiores dificuldades enfrentadas pelas mães e deve haver maior contribuição da equipe de saúde no sentido de orientar quanto à prática correta ${ }^{35}$. Para isso, é necessário que haja integração entre os profissionais, a fim de que todos sejam capacitados quanto às devidas orientações e cuidados, sejam médicos, nutricionistas, enfermeiros ou ACS.

Em se tratando dos benefícios que o AM pode trazer para a mãe e bebê, foi relatada a proteção às doenças $(93,6 \%)$, fortalecimento do vínculo afetivo (95\%), que o leite materno é um alimento completo $(84,4 \%)$, de fácil digestão $(90,1 \%) \mathrm{e}$ que faz o bebê crescer saudável (95\%). Os benefícios do AM são amplos e cada vez mais conhecidos, no entanto, ainda há uma tendência a relacionar essas vantagens apenas a saúde da criança, apesar de estudos mostrarem o impacto positivo também na saúde da mulher ${ }^{36}$. É importante que essas mães sejam orientadas quanto a essas vantagens a fim de encorajá-las a essa prática.

A primeira amamentação deve ocorrer imediatamente após o parto. Essa recomendação corresponde ao $4^{\circ}$ Passo da Iniciativa Hospital Amigo da Criança e é uma estratégia de promoção, proteção e apoio ao AM. Esse contato auxilia na interação da mãe com bebê e conseqüentemente fortalecimento do vínculo entre eles, aumentando a duração do AM e reduzindo mortalidade neonatal ${ }^{37,38}$. Como mostram os resul- 
tados, $85,1 \%$ das mulheres deste estudo tinham essa concepção.

Em relação ao intervalo de tempo que o leite materno deve ser oferecido, a influência da dieta e fatores que podem auxiliar ou dificultar o AM, a maioria das mães mostrou bom conhecimento teórico. No entanto, isso não se refletiu na prática do AME. Diversos fatores podem estar associados a esses resultados, dentre os quais a forma como essas informações são passadas às mães, uma vez que a prática do AM não deve ser encorajada apenas no pré-natal e maternidade, mas é preciso que haja uma educação continuada envolvendo essas mães, familiares e comunidade, já que o AM depende não só da decisão da mãe em fazê -lo, mas também de fatores socioculturais, onde muitas vezes informações errôneas são difundidas de geração a geração, prejudicando a prática do $\mathrm{AM}^{39,40}$.

Essa continuidade no acompanhamento da promoção do AM torna-se dificultosa diante do quantitativo de profissionais para demanda a ser atendida, considerando tanto os vinculados às UBS e, especialmente, os do Núcleo de Apoio a Saúde da Família (NASF). O tempo e a freqüência de consultas muitas vezes são escassos para haver uma orientação e manutenção efetivas. Esses e outros fatores comprometem o acompanhamento integral à saúde da mãe e da criança. É preciso que haja planejamento entre os diversos setores (saúde, educação e trabalho) a fim de propor melhor condição de promoção à saúde. Neste contexto, deve-se enfatizar a importância da equipe multiprofissional na promoção da saúde, com ênfase na atuação interdisciplinar, através da sensibilização da população quanto à adoção de hábitos mais saudáveis desde o início da vida.

\section{Considerações finais}

As mães mostraram bom conhecimento sobre assuntos relacionados ao AM, no entanto, esses conhecimentos não se refletiram na prática. As prevalências de AME e AMT das crianças estão abaixo das recomendações do Ministério da Saúde e OMS. É necessário que haja planejamento e articulação de ações que visem à promoção, proteção e apoio ao AM nessa população. Para isto, é importante uma rede de apoio, com a participação de toda a família, em especial àqueles mais presentes na rotina da mulher e criança e a atuação da equipe multiprofissional de saúde de forma interdisciplinar, apoiando e auxiliado mãe, família e criança para um processo de AM mais tranquilo e bem-sucedido, desde o pré-natal. Deve-se considerar a cobertura dos profissionais na área, pois, se insuficiente, gera demandas que dificultam o acompanhamento integral e de qualidade. 


\section{Colaboradores}

EM Santos trabalhou na elaboração do projeto, revisão da literatura, coleta, tabulação e interpretação dos dados, e digitação do artigo. LS Silva trabalhou na elaboração do projeto, confecção do questionário para pesquisa e na coleta e tabulação dos dados. BFS Rodrigues trabalhou na coleta de dados. TMAX Amorim trabalhou na digitação do artigo, bem como a tradução para o inglês. CS Silva trabalhou na revisão bibliográfica. JMC Borba trabalhou na revisão da literatura e correção do texto. FCLP Tavares trabalhou na elaboração do projeto, confecção de questionários, treinamento, tabulação e interpretação dos resultados, análise estatística, correção do texto e da bibliografia.

\section{Referências}

1. Victora CG, Horta BL, Loret de Mola C, Quevedo L, Pinheiro RT, Gigante DP, Gonçalves H, Barros FC. Association between breastfeeding and intelligence, educational attainment, and income at 30 years of age: a prospective birth cohort study from Brazil. Lancet Glob Health 2015; 3(4):199-205.

2. Brasil. Ministério da Saúde (MS). II Pesquisa de prevalência de aleitamento materno nas capitais brasileiras e Distrito Federal. Brasília: MS; 2009.

3. Lopes TSP, Moura LFAD, Lima MCMP. Association between breastfeeding and breathing pattern in children: a sectional study. J Pediatr 2014; 90(4):396-402.

4. Souza SL, Castro RM, Nogueira MI. Comportamento alimentar neonatal. Rev Bras Saúde Matern Infant 2003; 3(3):241-246.

5. Fonseca-Machado MO, Haas VJ, Stefanello J, Nakano MAS, Gomes-Sponholz F. Breastfeeding: knowledge and practice. Rev Esc Enferm USP 2012; 46(4):809-815.

6. Takahashi K, Ganchimeg T, Ota E, Volgel JP, Souza JP, Laopaiboon M, Castro CP, Jayaratne K, Ortiz-Panozo E, Lumbiganon P, Mori R. Prevalence of early initiation of breastfeeding and determinants of delayed initiation of breastfeeding: secondary analysis of the WHO Global Survey. Scientific Reports 2017; 7:1-10.

7. Gupta PM, Perrine CG, Chen J, Elam-Evans LD, Flores-Ayala R. Monitoring the World Health Organization Global Target 2025 for Exclusive Breastfeeding: Experience From the United States. J Hum Lact 2017; 33(3):578-581.

8. Carvalho MR, Tavares LAM. Amamentação: bases científicas. Rio de Janeiro: Guanabara Koogan; 2010.

9. Caminha MFC, Batista Filho M, Serva VB, Arruda IKG, Figueiroa JN, Lira PIC. Tendências temporais e fatores associados à duração do aleitamento materno em Pernambuco. Rev Saude Publica 2010; 44(2):240-248.

10. Venâncio SI, Escuder MM, Saldivas SRDM, Giugliani ERJ. A Prática do aleitamento materno nas capitais brasileiras e Distrito Federal: Situação atual e avanços. J Pediatr 2010; 86(4):317-324.

11. Maranhão TA, Gomes KRO, Nunes LB, Moura, LNB. Fatores associados ao aleitamento materno exclusivo entre mães adolescentes. Cad. Saúde Colet 2015; 23(2):132-139.

12. Guimarães CMS, Conde RG, Brito BC, Gomes-Sponholz FA, Oriá MOB, Monteiro JCS. Comparação da autoeficácia na amamentação entre puérperas adolescentes e adultas em uma maternidade de Ribeirão Preto, Brasil. Texto Contexto Enferm 2017; 26(1):1-9.

13. Salustiano LPQ, Diniz ALD, Abdallah VOS, Pinto RMC. Fatores associados à duração do aleitamento materno em crianças menores de seis meses. Rev Bras Ginecol Obstet 2012; 34(1):28-33.

14. Figueredo SF, Mattar MJG, Abrão ACFV. Hospital Amigo da Criança: prevalência de aleitamento materno exclusivo aos seis meses e fatores intervenientes. Rev Esc Enferm. USP 2013; 47(6):1291-1297.

15. Damião JJ. Influência da escolaridade e do trabalho maternos no aleitamento materno exclusivo. Rev Bras Epidemiol 2008; 11(3):442-452.

16. Borges AL, Philippi ST. Opinião de mulheres de uma unidade de saúde da família sobre a quantidade de leite materno produzido. Rev Latino-Am Enfermagem 2003; 11(3):287-292. 
17. Souza MHN, Sodré VRD, Silva FNF. Prevalência e fatores associados à prática da amamentação de crianças que frequentam uma creche comunitária. Cienc. Enferm 2015; 21(1):55-67.

18. Brant PMC, Affonso HS, Vargas LC. Incentivo à amamentação exclusiva na perspectiva das puérperas. Cogitare Enferm 2009; 14(3):512-7.

19. Machado MCM, Assis KF, Oliveira FCC, Ribeiro AQ, Araújo RMA, Cury AF, Priore SE, Franceschini SCC. Determinantes do abandono do aleitamento materno exclusivo: fatores psicossociais. Rev Saude Publica 2014; 48(6):985-994.

20. Carrascoza KC, Possobon RF, Ambrosano GMB, Júnior ALC, Moraes ABA. Determinantes do abandono do aleitamento materno exclusivo em crianças assistidas por programa interdisciplinar de promoção à amamentação. Cien Saude Colet 2011; 16(10):4139-4146.

21. Faleiros FTV, Trezza EMC, Carandina L. Aleitamento materno: fatores de influência na sua decisão e duração. Rev. Nutr 2006; 19(5):623-630.

22. Wenzel D, Souza SB. Fatores associados ao aleitamento materno nas diferentes Regiões do Brasil. Rev. Bras. Saúde Matern. Infant 2014; 14(3):241-249.

23. Boff ADG, Paniagua LM, Scherer S, Goulart BNG. Aspectos socioeconômicos e conhecimento de puérperas sobre o aleitamento materno. Audiol Commun Res 2015; 20(2):141-145.

24. Martins CC, Vieira GO, Vieira TO, Mendes CMC. Fatores de riscos maternos e de assistência ao parto para a interrupção precoce do aleitamento materno exclusivo: estudo de coorte. Feira de Santana: Rev Baiana de Saúde Publica 2011; 35(1):167-178.

25. Ramos VW, Ramos JW. Aleitamento materno, desmame e fatores associados. CERES: Nutrição \& saúde 2007; 2(1):43-50.

26. Brasil. Ministério da Saúde (MS). Programa de humanização no pré-natal e nascimento. Brasília: MS; 2002.

27. Pérez-Escamilla R, Lutter C, Segall AM, Rivera A, Treviño-Siller S, Sanghvi T. Exclusive breast-feeding duration is associated with attitudinal, socioeconomic and biocultural determinants in three Latin American countries. J Nutr 1995; 125(12):2972-2984.

28. Parizoto GM, Parada CMGL, Venâncio SI, Carvalhaes MABL. Tendência e determinantes do aleitamento materno exclusivo em crianças menores de 6 meses. $J$ Pediatr 2009; 85(3):201-208.

29. Siqueira C, De Carvalho M, Couto M, Godoi A. Fatores associados à amamentação na primeira hora de vida. Rev Saude Publica 2011; 45(1):69-78.

30. Carrascoza KC, Possobon RF, Ambrosano GMB, Júnior ALC, Moraes ABA. Fatores determinantes do uso de chupeta entre crianças participantes de programa de incentivo ao aleitamento materno. Rev. CEFAC 2014; 16(2):582-591.
31. Neu AP, Silva AMT, Mezzomo CL, Busanello-Stella AR. Aleitamento: Relação com hábitos de sucção e aspectos socioeconômicos familiares. Rev. CEFAC 2014; 16(3):883-891.

32. Pellegrinelli ALR, Pereira SCL, Ribeiro IP, Santos LC. Influência do uso de chupeta e mamadeira no aleitamento materno exclusivo entre mães atendidas em um Banco de Leite Humano. Rev. Nutr 2015; 28(6):631639.

33. Escarce AG, Araújo NG, Friche AAL, Motta AR. Influência da orientação sobre aleitamento materno no comportamento das usuárias de um hospital universitário. Rev. CEFAC 2013; 15(6):1570-1582.

34. Pontes AM, Lucena KDT, Silva ATMC, Almeida LR, Deininger LSC. As repercussões do aleitamento materno exclusivo em crianças com baixo peso ao nascer. Saúde em Debate 2013; 37(97):354-361.

35. Brasil. Ministério da Saúde (MS). Pré-natal e puerpério: atenção qualificada e humanizada - manual técnico. Brasília: MS; 2006.

36. Rea MF. Os benefícios da amamentação para a saúde da mulher. J Pediatr 2004; 80(5):142-146.

37. Boccolini CS, Carvalho ML, Oliveira MIC, Vasconcellos AGG. Fatores associados à amamentação na primeira hora de vida. Rev Saude Publica 2011; 45(1):69-78.

38. Belo MNM, Azevedo PTACC, Belo MPM, Serva VMSBD, Filho MB, Figueiroa JN, Caminha MFC. Aleitamento materno na primeira hora de vida em um Hospital Amigo da Criança: prevalência, fatores associados e razões para sua não ocorrência. Rev. Bras. Saúde Matern. Infant 2014; 14(1):65-72.

39. Marques ES, Cotta RMM, Priore SE. Mitos e crenças sobre o aleitamento materno. Cien Saude Colet 2011; 16(5):2461-2468.

40. Andrade RD, Santos JS, Maia MAC, Mello DF. Fatores relacionados à saúde da mulher no puerpério e repercussões na saúde da criança. Esc Anna Nery 2015; 19(1):181-186.

Artigo apresentado em 08/12/2016

Aprovado em 02/07/2017

Versão final apresentada em 04/07/2017 